\title{
AMBIENT TEMPERATURE-GRADIENT COMPENSATED LOW-DRIFT THERMOPILE FLOW SENSOR
}

\author{
M. Dijkstra, T.S.J. Lammerink, M.J. de Boer, R.J. Wiegerink and M. Elwenspoek \\ $\mathrm{MESA}^{+}$Institute for Nanotechnology, University of Twente, The Netherlands
}

\begin{abstract}
A highly-sensitive thermal flow sensor for liquid flow with $\mathrm{nl} \cdot \mathrm{min}^{-1}$ resolution has been realised. The sensor consists of freely-suspended silicon-rich silicon-nitride microchannels with integrated $\mathrm{Al}$ heater resistors and $\mathrm{Al} /$ poly-Si ${ }^{++}$thermopiles. The influence of drift in the thin-film metal resistors is effectively eliminated by using thermopiles combined with an adequate measurement method, where the power in the heater resistors is controlled, e.g. constant-power calorimetric method or temperature balancing method. The special meandering layout of the microchannels and the placement of thermopile junctions increases sensitivity by summing the thermopile voltages due to convection by fluid flow, whereas the influence of ambient temperature gradients is compensated for.
\end{abstract}

\section{INTRODUCTION}

The miniaturisation of microfluidic components asks for accurate and reliable measurement of tiny fluid flow rates in the order of $\mathrm{nl} \cdot \mathrm{min}^{-1}$. Current micromechanical thermal flow sensors are capable of measuring down to $\mathrm{nl} \cdot \mathrm{min}^{-1}$ resolution [1-4]. Important problems limiting the accuracy of these thermal flow sensors are: the drift in the electrical resistance of thin-film layers used for heating and temperature sensing, and the influence of external temperature gradients across the sensor chip. Thermistors used for temperature sensing can be avoided, by using thermopiles to measure a temperature difference, with inherent zero-offset [5-6]. Using thermopiles, the flow sensor can be made independent of resistance drift by applying power control on heater resistors. Additionally, a temperature-balancing feedback loop can be used to compensate for thermopile sensitivity drift [6].

This paper presents a low-drift calorimetric flow sensor for liquid flows with $\mathrm{nl} \cdot \mathrm{min}^{-1}$ resolution. The flow sensor consists of freely-suspended microchannels with integrated thermopiles for temperature sensing, with power control applied on heater resistors used. Furthermore, a special meandering microchannel layout is utilised to compensate for the influence of external temperature gradients across the sensor chip.

\section{COMPENSATION CONCEPT}

Ambient temperature-gradients are compensated using a meandering microchannel layout, in which a fluid flow $Q$ passes in alternating direction through several freely-suspended microchannels used for thermal flow sensing (Figure 1). Thermopiles are connected in series and positioned on the freely suspended microchannels in such a way that each thermopile measures a temperature difference $\Delta T$ between an upstream junction temperature $T_{u p}$ and a downstream junction temperature $T_{\text {down }}$.

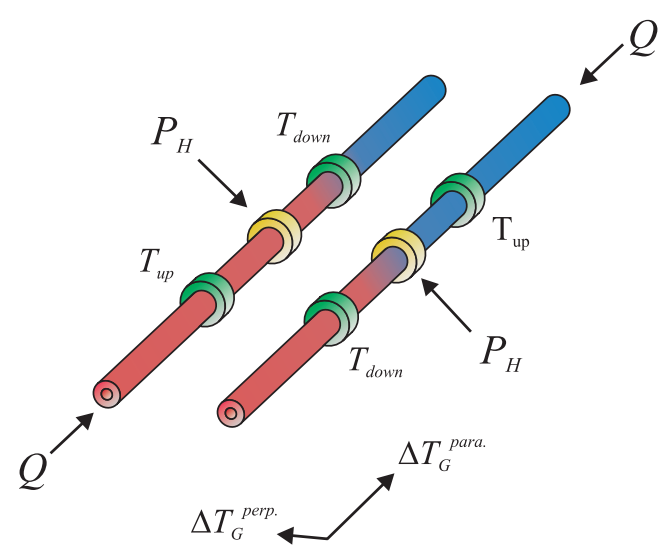

Figure 1: Ambient temperature-gradient compensated flow sensor concept.

Effectively the flow passes the same sensor configuration several times, while flowing through the meandering microchannel, giving a summation of multiple thermopile voltages due to convection by fluid flow.

External temperature gradients $\Delta T_{G}$ of first order can be decomposed in two directions. A temperature gradient parallel to the microchannels $\Delta T_{G}{ }^{\text {para. }}$ changes $T_{u p}$ and $T_{\text {down }}$ in opposite ways on alternating microchannels, not influencing the total measured $\Delta T$. A gradient perpendicular to the microchannels $\Delta T_{G}{ }^{\text {perp. }}$ changes the offset temperature of each microchannel, not influencing the difference between $T_{u p}$ and $T_{\text {down }}$ on the same microchannel. First order temperature gradients can therefore be fully compensated. However, the heater resistors are slightly temperature dependent and therefore require control of the heating power $P_{H}$, in order for the compensation concept to work properly.

\section{SENSOR FABRICATION}

The fabricated thermopile flow sensor uses freelysuspended surface microchannels for on-chip transport of fluid. The microchannels are fabricated without the requirement for sacrifical layer etching and allow for the integration of sensor elements in close proximity to the fluid [7]. The technology has been applied in the fabrication of highly-sensitive flow sensors $[4,6,8]$.

Figure 2 gives a schematic overview of the process scheme for the fabrication of the thermopile flow sensor. Surface microchannels are created by isotropic dry etching, using high-density $\mathrm{SF}_{6}$ plasma with zero selfbias, through etch holes $2 \mu \mathrm{m}$ in width, in a low-stress $500 \mathrm{~nm}$ silicon-rich silicon-nitride (SiRN) layer. The etch holes and inner surfaces of the microchannels are conformally coated by a second low-stress LPCVD deposited $1.3 \mu \mathrm{m}$ SiRN layer, resulting in completely sealed microchannels, while leaving a planar substrate 

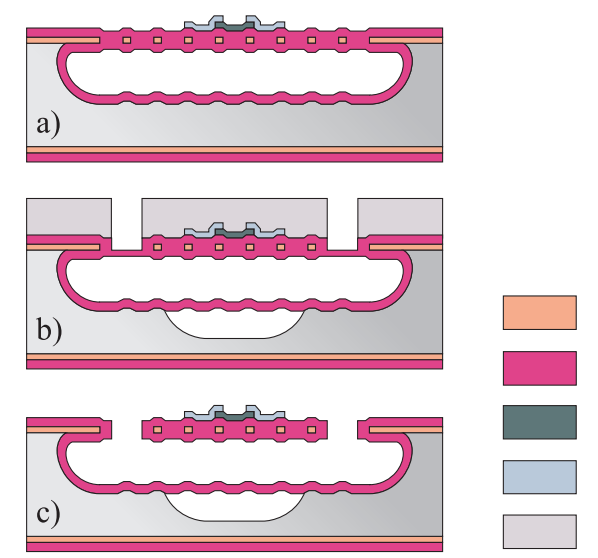

$500 \mathrm{~nm}$ SiRN

$1.3 \mu \mathrm{m} \mathrm{SiRN}$

$200 \mathrm{~nm}$ poly-Si

$200 \mathrm{~nm} \mathrm{Al}$

photoresist

Figure 2: Process scheme for the fabrication of the temperature-gradient compensated thermopile flow sensor.

surface for the integration of $\mathrm{Al} / \mathrm{poly}-\mathrm{Si}^{++}$thermopiles and Al heater resistors. The thermopiles are created by LPCVD deposition and boron doping, by solid source diffusion, of a $200 \mathrm{~nm}$ poly-Si ${ }^{++}$layer and sputtering of a $200 \mathrm{~nm} \mathrm{Al} \mathrm{layer} \mathrm{(Figure} \mathrm{2a).} \mathrm{The} \mathrm{surface} \mathrm{microchannels}$ are released by $\mathrm{SF}_{6}$ plasma etching for thermal isolation from the heat-conducting substrate, with the photoresist mask protecting the sensor elements during the release (Figure 2b). The photoresist is removed after fluidic entrance holes are etched through the SiRN layer (Figure 2c).

Figure 3 shows a micrograph of the fabricated temperature-gradient compensated flow sensor. The sensor contains three parallel $20 \mu \mathrm{m}$ diameter microchannels, which cross a thermal-isolation cavity $(1.2 \times 2.0 \mathrm{~mm}, 250 \mu \mathrm{m}$ deep) several times in alternating flow directions. Heater resistors are centered on the freely suspended microchannels and connected in series. Fourpoint contact is used for accurate power control.

Figure 4 shows a close-up of the surface microchannels with integrated $\mathrm{Al} /$ poly- $\mathrm{Si}^{++}$thermopiles having eight junctions on either side, for measuring the down- and upstream temperature difference.

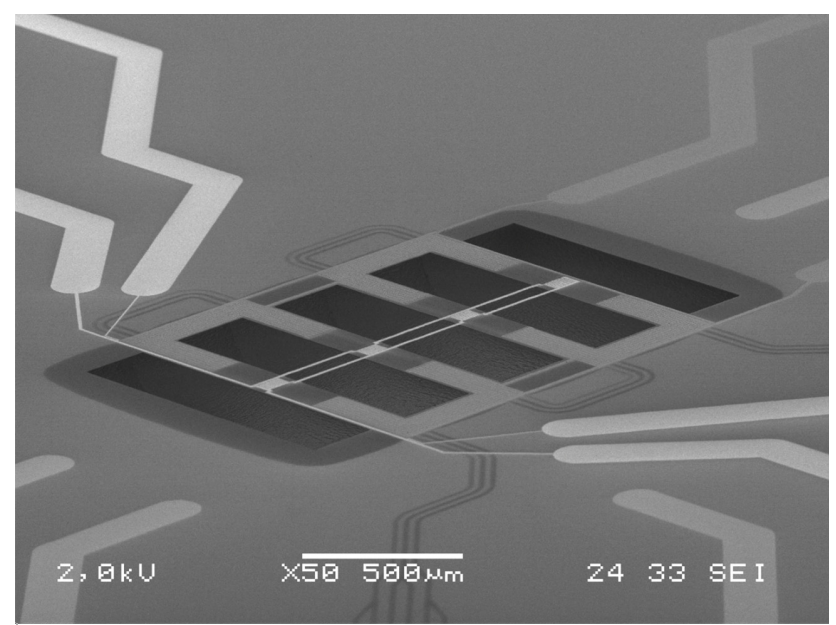

Figure 3: Microchannel temperature-gradient compensated thermopile flow sensor.

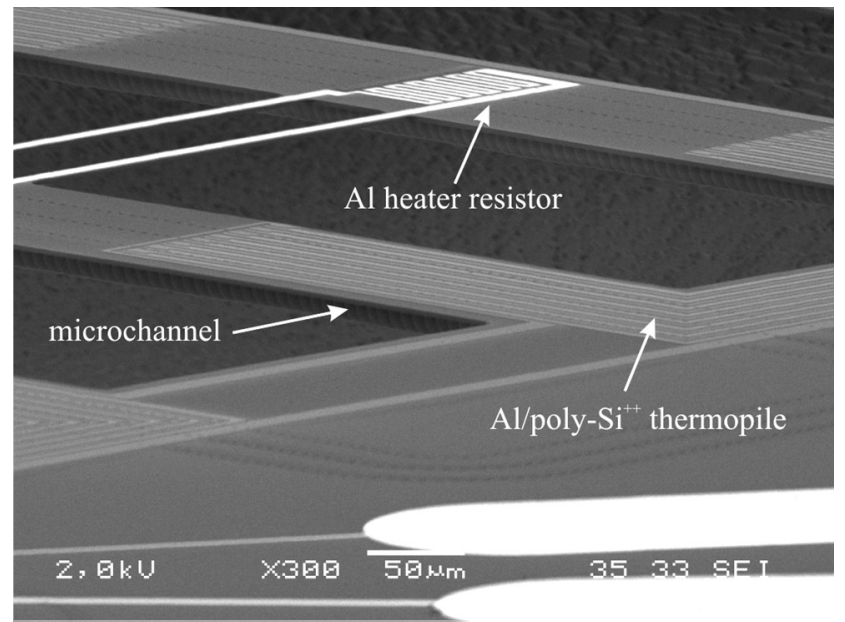

Figure 4: Close-up of the microchannels with integrated Al heater resistors and Al/poly-Si ${ }^{++}$thermopiles, suspended over a thermal isolation cavity etched in the substrate.

\section{SENSOR MODELLING}

A thermal model of the flow sensor was constructed in COMSOL Multiphysics. The temperature field was solved including the thermal conduction in the flow sensor and surrounding air and forced convection by water flow through the microchannels. The flow inside the microchannels is approximated using a fixed velocity profile corresponding to a given flow rate, without affecting the accuracy of the thermal model. Additionally, deposited layers are modelled as highly conductive layers, including the thermopiles and heater resistors.

Figure 5 shows isothermal surfaces of the resulting temperature field at $350 \mathrm{nl} \cdot \mathrm{min}^{-1}$ water flow through the microchannels, with $0.6 \mathrm{~mW}$ total heating power applied. Clearly, the effect of forced convection by the alternating direction of the flow through the microchannels can be observed at this relatively high flow rate. The high

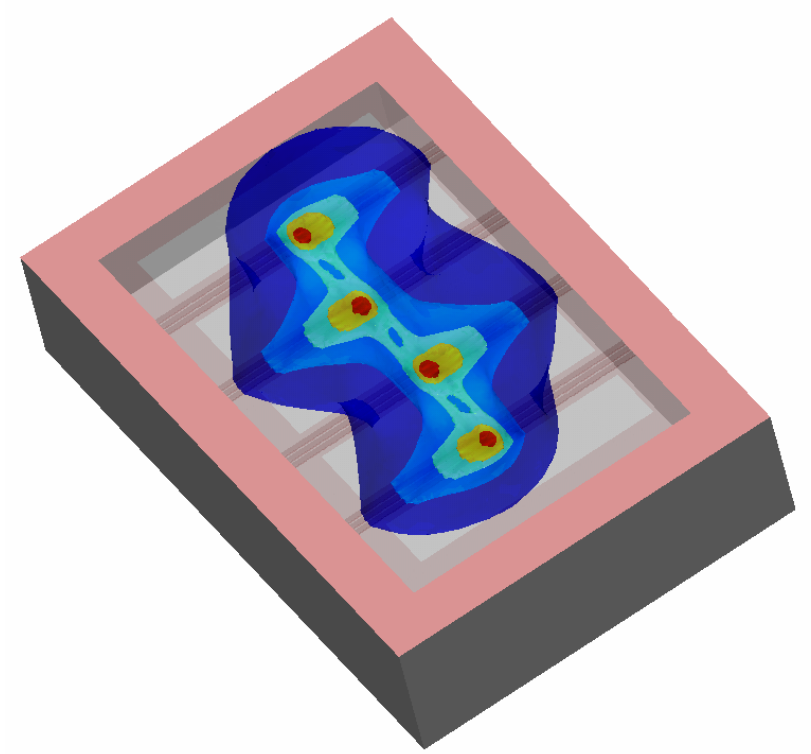

Figure 5: Thermal FEM model of the flow sensor, showing the influence of convection by the alternating direction of $350 \mathrm{nl} \cdot \mathrm{min}^{-1}$ water flow through the microchannels at $0.6 \mathrm{~mW}$ total heating power. 


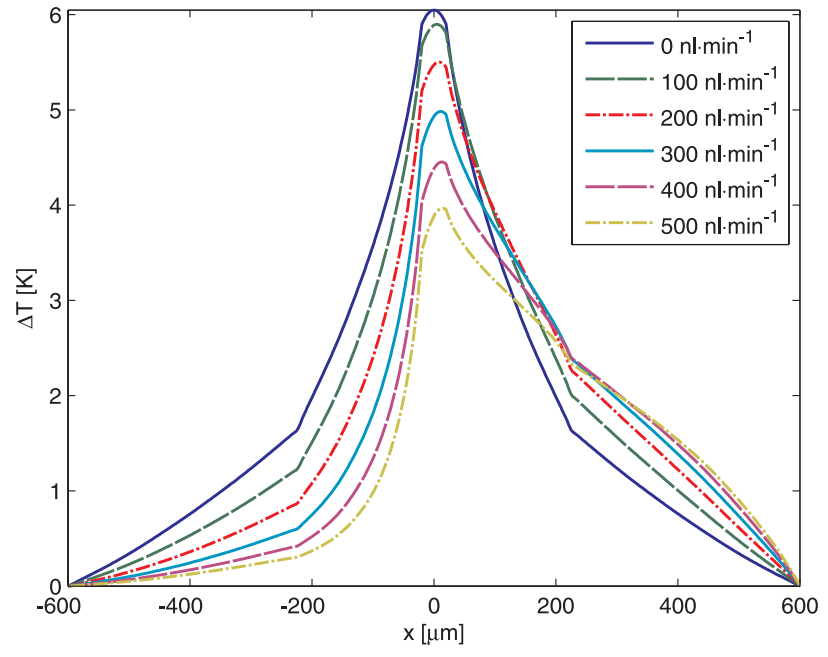

Figure 6: Temperature profiles for various water flow rates in the positive $x$-direction along a microchannel suspended at $\pm 600 \mu \mathrm{m}$, with $0.6 \mathrm{~mW}$ total heating power applied.

thermal conduction of the Al leads connecting the heater resistors is also apparent. Although, there is no heat flow between adjacent microchannels for the temperature field by conduction alone.

Figure 6 shows temperature profiles along the microchannel at various water flow rates. A difference in the slope of the temperature profiles can be observed at $\pm 225 \mu \mathrm{m}$ from the centre, where the thermopile junctions are situated on the microchannel, with the thermopile leads causing much higher thermal conduction to the substrate.

\section{EXPIREMENTAL RESULTS}

\section{Flow Rate Sensitivity}

The fabricated thermopile flow sensors chips $(12.5 \times$ $12.5 \mathrm{~mm}$ ) have bondpads and microchannel entrance holes on fixed positions for self alignment with a chip holder (Figure 7). The chip holder contains O-rings for fluidic interfacing and pogo-pins for electrical connections to the sensor chip. Water flow is applied by an elevation head $\Delta h$, giving a stable flow rate. The flow rate is calibrated by microbalance weighing, where evaporation from the balance, in the order of $100 \mathrm{nl} \cdot \mathrm{min}^{-1}$, has to be taken into consideration. A source measurement unit (Keithley 2420) was used for controlling a fixed total heating power $P_{H}$. The thermopile voltage $V_{T C}$ was obtained by a nano-volt meter (HP 34420A).

Figure 8 shows the thermopile voltage $V_{T C}$ dependency on water flow rate $Q$ up to $550 \mathrm{nl} \cdot \mathrm{min}^{-1}$ at various applied heating powers. The sensor shows linear sensitivity up to flow rates of about $100 \mathrm{nl} \cdot \mathrm{min}^{-1}$. The heater temperature decreases at higher flow rates (Figure 6), causing the sensor output to decrease according to King's law. The dashed lines in figure 8 show fitted FEM results after scaling calculated temperature differences to measured voltages. Measurements show offsets in the thermopile voltage, which are dependent on the heating power and can be attributed to sensor asymmetries.

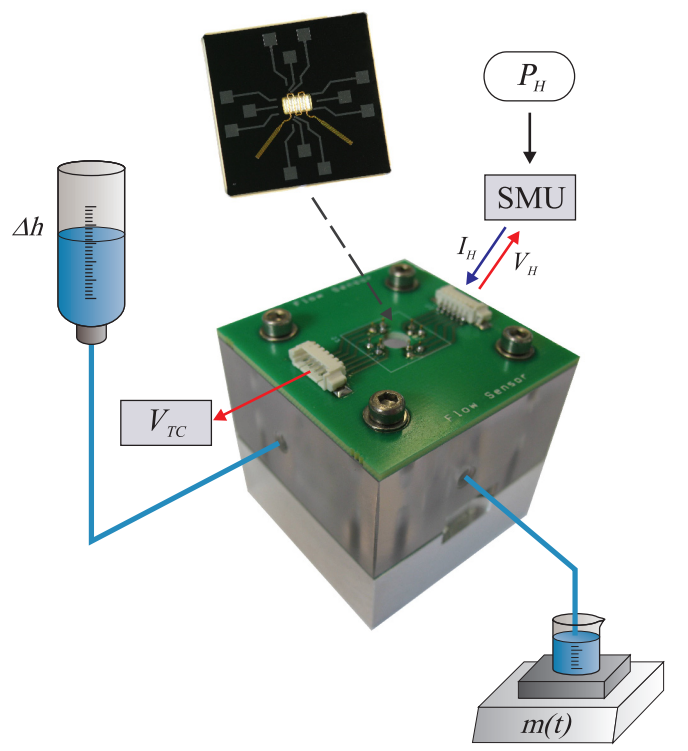

Figure 7: Overview of the flow measurement setup, with the chip holder establishing fluidic and electrical connections.

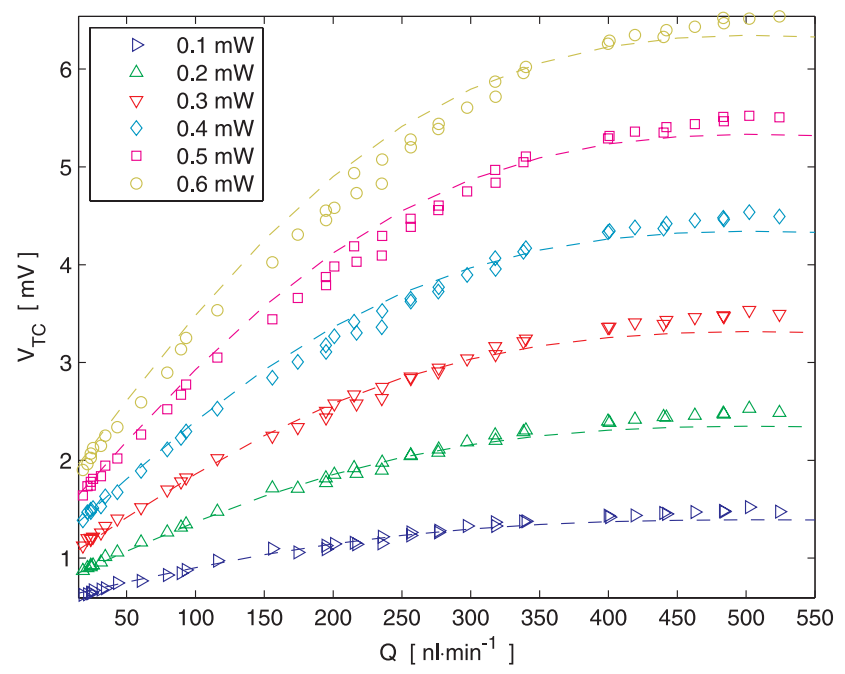

Figure 8: Flow sensor thermopile-voltage $V_{T C}$ dependency on water flow rate $Q$ at various applied total heating powers. Dashed lines show fitted FEM results.

\section{Ambient Temperature-Gradient Sensitivity}

A temperature-gradient measurement setup was constructed in order to determine the influence of external temperature gradients on the thermopile voltage (Figure 9). The setup consists of a $5 \mathrm{~mm}$ thick $\mathrm{Cu}$ plate, which is heated from one side by a resistor dissipating $25 \mathrm{~W}$, while the other side is connected to a large aluminium plate acting as heat sink, thus creating a well defined temperature gradient. The temperature gradient was determined to be approximately $2.8 \mathrm{~K} \cdot \mathrm{cm}^{-1}$, measured using two Pt-100 elements placed $10 \mathrm{~cm}$ apart. The sensor chip is placed in the centre of the $\mathrm{Cu}$ plate and probes are used to measure the thermopile voltage using a nano-volt meter (HP 34420A). This allows the sensor chip to be rotated and the influence of the external temperature gradient to be measured at any angle. 


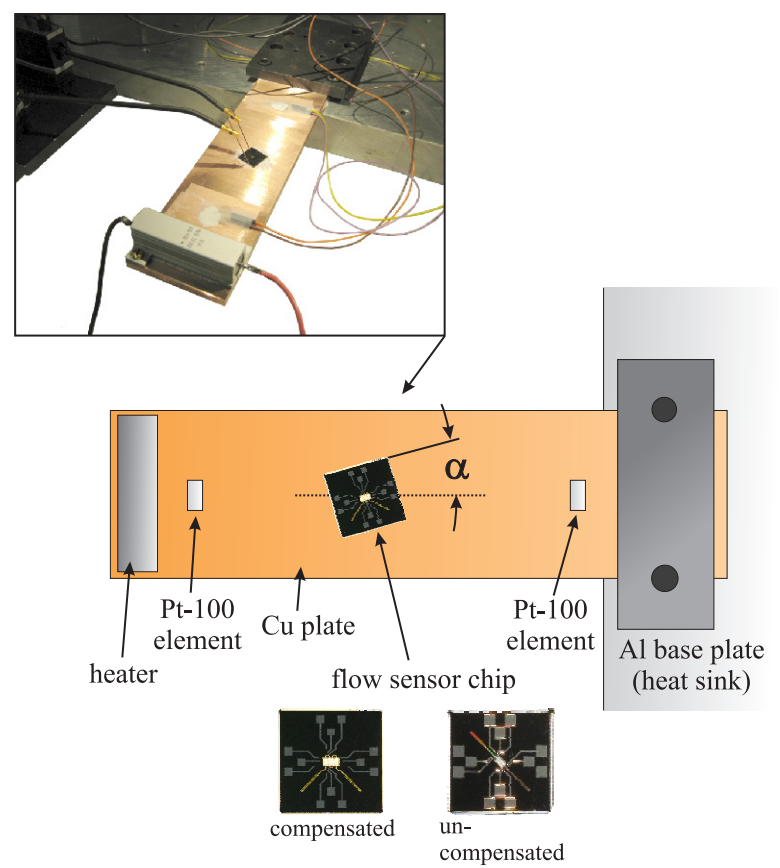

Figure 9: Temperature-gradient measurement setup for inducing a well defined temperature gradient across the sensor chip.

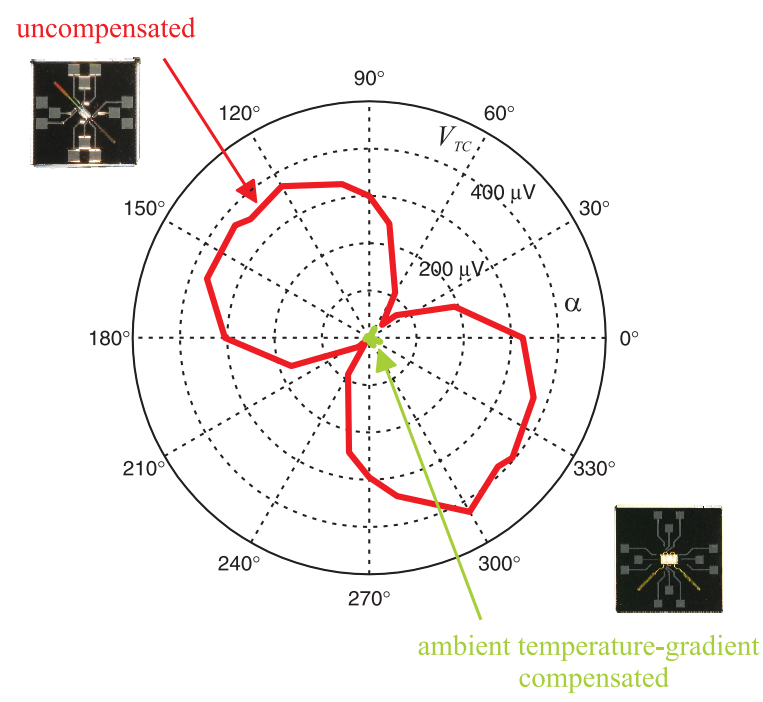

Figure 10: External temperature-gradient sensitivity of the thermopile voltage $V_{T C}$ measured for a compensated sensor and a single-microchannel uncompensated flow sensor.

An ambient temperature-gradient compensated flow sensor and an uncompensated single-microchannel flow sensor, for comparison, were measured, with both sensors having nearly equal amounts of thermopile junctions. The ambient temperature-gradient compensated flow sensor shows to be completely compensated for external temperature gradients (Figure 10). The uncompensated flow sensor shows a figure-of-eight sensitivity pattern, because the flow sensor is only sensitive to temperature gradients parallel to the microchannel $\Delta T^{\text {para. }}$.

\section{CONCLUSIONS}

A calorimetric flow sensor measuring liquid flow down to $\mathrm{nl} \cdot \mathrm{min}^{-1}$ resolution has been realised, using freely-suspended microchannels for thermal isolation form the silicon substrate. The flow sensor shows a linear response for water flow up to $100 \mathrm{nl} \cdot \mathrm{min}^{-1}$, which is accurately described by a thermal FEM model of the sensor.

Influence of drift in thin-film metal resistors is effectively eliminated by using power control, in conjunction with thermopiles for temperature sensing. It is demonstrated that this measurement method in combination with a special meandering microchannel layout can effectively compensate drift by ambient temperature-gradients across the sensor chip. Compared to a single microchannel uncompensated flow sensor a significant improvement is obtained in sensor accuracy.

\section{ACKNOWLEDGEMENT}

The authors would like to thank the Dutch Technology Foundation (STW) for their financial support through the low-drift micro-flowsensor project (TET.6634).

\section{REFERENCES}

[1] Y. Mizuno, M. Liger, Y-C. Tai, "Nanofluidic Flowmeter using Carbon Sensing Element", Proc. IEEE MEMS, 2004, pp. 322-325.

[2] S. Wu, Q. Lin, Y. Yuen, Y-C. Tai, "MEMS flow sensor for nano-fluidic applications", Sensors and Actuators A, vol. 89, pp. 152-158, 2001.

[3] H. Ernst, A. Jachimowicz, G.A. Urban, "High resolution flow characterization in Bio-MEMS", Sensors and Actuators A, vol. 100, pp. 54-62, 2002.

[4] M. Dijkstra, M.J. de Boer, J.W. Berenschot, T.S.J. Lammerink， R.J. Wiegerink，M. Elwenspoek, "Miniaturized Thermal Flow Sensor with PlanarIntegrated Sensor Structures on Semicircular Surface Channels", Sensors and Actuators A, vol. 143, pp. 1-6, 2008.

[5] P. Brushi, A. Diligente, D. Navarrini, M. Piotto, "A Double Heater Integrated Gas Flow Sensor with Thermal Feedback", Sensors and Actuators A, vol. 123-124, pp. 210-215, 2005.

[6] M. Dijkstra, T.S.J. Lammerink, M.J. de Boer, J.W. Berenschot, R.J. Wiegerink, M. Elwenspoek, "Low-Drift Flow Sensor with Zero-Offset Thermopile-Based Power Feedback", Proc. DTIP, 2008.

[7] M. Dijkstra, M.J. de Boer, J.W. Berenschot, T.S.J. Lammerink， R.J. Wiegerink，M. Elwenspoek, "A Versatile Surface Channel Concept for Microfluidic Applications", J. Micromech. Microeng., vol. 17, pp. 1971-1977, 2007.

[8] J. Haneveld, T.S.J. Lammerink, M. Dijkstra, H. Droogendijk, M.J. de Boer, R.J. Wiegerink, "Highly Sensitive Micro Coriolis Mass Flow Sensor", Proc. IEEE MEMS, 2008, pp. 920-923. 